Type of the Paper Article

\title{
Oleanolic Acid Induces p53 Dependent Apoptosis via the ERK/JNK/AKT Pathway in Cancer Cell Lines
}

\author{
Gyeong-Ji Kim¹, Hyeon-Ju Jo², Kang-Hyun Chung'2, Kwon-Jai Lee², and Jeung Hee An"* \\ ${ }^{1}$ Division of Food Bioscience, Konkuk University, Chunju 27478, Korea; E-mail: kgj8495@hanmail.net (G.-J.K); \\ anjhee@hanmail.net (J.H.A) \\ 2Department of Food Science and Technology, Seoul National University of Science \& Technology, Seoul \\ 139-743, Korea; E-mail: pomrin@hanmail.net (H.-J.J); carl@seoultech.ac.kr (K.H.J) \\ ${ }^{3}$ Department of Advanced Materials Engineering, Daejeon University, Daejeon 300-716, Korea; E-mail: \\ jmul@ssu.ac.kr \\ * Correspondence: anjhee@hanmail.net; Tel.: +82-43-840-3584
}

\begin{abstract}
We evaluated oleanolic acid (OA)-induced anti-cancer activity, apoptotic mechanism, cell cycle status, and MAPK kinase signaling in DU145 (prostate cancer), MCF-7 (breast cancer), and U87 (human glioblastoma) cells. The IC50 values for OA-induced cytotoxicity were 112.57 in DU145, 132.29 in MCF-7, and 163.60 in U87 cells, respectively. OA (at $100 \mu \mathrm{g} / \mathrm{mL}$ ) increased the number of apoptotic cells to $27.0 \%$ in DU145, $27.0 \%$ in MCF-7, and $15.7 \%$ in U87 cells, when compared to control cells. This enhanced apoptosis was due to increases in p53, cytochrome c, and Bax expression. OA-treated DU145 cells were arrested in G2 because of the activation of p-ERK and p21, and the decrease in cyclin B1 and cyclin E expression. Furthermore, OA-treated MCF-7 cells were arrested in G1 owing to the activation of p-JNK, p-ERK, p21, and p27, and the decrease in p-AKT, cyclin E, and CDK2. U87 cells also exhibited G1 phase arrest caused by the increase in p-ERK, p-JNK, p21, and p27, and the decrease in CDK2. Thus, OA arrests the cell cycle in different phases, and increases apoptosis in cancer cells. These results suggest that OA may alter the expression of cell cycle regulatory proteins in a cancer type-dependent manner.
\end{abstract}

Keywords: anticancer activity; apoptosis; oleanolic acid; cell cycle arrest; mapk signaling

\section{Introduction}

Cancer represents one of the major health problems [1]. In 2008, approximately 12.7 million cancers were reported worldwide, and this number is estimated to increase to 21 million in 2030 [2]. According to the National Cancer Information Center of Korea, cancer is more common in males compared to females [3]. In 2014, the cancer incidence in Korea was highest for thyroid cancer, followed by stomach, colon, breast, lung, and prostate cancers [3]. Chemotherapy is effective against most cancer types, but drug resistance limits the success of treatment in most cases, while the inability of the drug to distinguish between normal and cancerous cells hinders its applicability [4-5]. Thus, utmost attention is being given to the search for better and safer drugs of natural origin, which may raise the efficacy of cancer treatment [5].

Apoptosis, the process of programmed cell death, is the most common mechanism for inducing death in cancer cells using targeted chemotherapy [6]. It is a regulated process of cell death, which is activated by stressors such as DNA damage, cytokines, and oxidative stress [7]. The p53 tumor suppressor is activated by oncogene-or DNA damage- induced signaling pathways, and accelerate the transcription of several genes that are involved in apoptosis, proapoptotic members of the Bcl-2 family and including those encoding for death receptors. [8]. Bax is an important proapoptotic member of the Bcl-2 family of proteins that regulates the balance between cellular survival and death [9]. In response to apoptosis signals, Bax is transformed into a fatal mitochondrial oligomer that causes mitochondrial damage; this represents an important step for the intrinsic apoptosis pathway $[10,11]$. In addition, the p53-induced apoptosis induces the activation of caspases [8]. Caspase 
activation primarily occurs through the activation of the death receptor pathway or through mitochondrial membrane depolarization [6].

The relationship between the cell cycle and apoptosis is underscored by the role of the p53 tumor suppressor gene, and those of the p21WAF1/CIP1 and BAX genes, which induce cell cycle arrest and cell death [12]. Cell proliferation is mediated by several signaling molecules and checkpoints (CDKs) that regulate cell division [13]. The progression through the cell cycle is positively regulated by cyclin $\mathrm{E}$ and the cyclin-dependent kinase (CDK) complex, which phosphorylate the retinoblastoma tumor suppressor protein to induce the transition from the G1 to the $S$ phase [10]. The p21WAF1/CIP1 and p27KIP1 kinase inhibitor proteins bind to the cyclin E/CDK2 complex and block the G1/S transition [14]. Besides these proteins, cyclin B1 plays a key role in the cell cycle transition from the G2 to M phase [15]. The decreased expression of cyclin B1 may disrupt cell growth, and promote malignant transcription [16].

Oleanolic acid (3- $\beta$-hydroxy-olea-12-en-28-oic acid, OA) is natural pentacyclic triterpenoic acid $[17,18]$. OA has chemopreventive, including hepatoprotective, tumor-suppressive, anti-inflammatory, various biological effects, contraceptive, anti-oxidant, and antineoplastic activities [19 - 21]. Anti-cancer activity of OA has been demonstrated in several cancer cell lines, such as KB, HT29, MCF-7, HONE-1, Hep-G2 and HL-60 [20, 22]. Recently, several reports have shown that OA can induce G1 cell cycle arrest in the GBC-SD, NOZ, HCT15, and K562 cell lines [21, 23]. Moreover, it was reported that OA induces a concentration-dependent $\mathrm{S}$ phase and G2/M phase cell cycle arrest in Panc-28 and Hep-G2 cells [24, 25]. The inhibitory effects of OA are due to the suppression of specific intracellular signaling pathways, such as the STAT3, JNK, AKT, and NF-kappaB signaling pathways [26] These studies indicate that OA can be proposed as an adjunct to cancer chemotherapy.

In this study, we investigated the cellular viability, apoptosis, and cell cycle in OA-treated DU145 (prostate cancer), MCF-7 (breast cancer), and U87 (human glioblastoma) cells. In addition, we examined the protein expression in relationship to apoptosis, cell cycle, and kinase signaling in these OA-treated cells. These results suggest that OA may alter the expression of cell cycle regulatory proteins in a cancer type-dependent manner.

\section{Materials and Methods}

\subsection{Cell culture and reagents}

Human prostate cancer (DU145), breast cancer (MCF-7), and glioblastoma cancer (U87) cell lines were purchased from the Korea Cell Line Bank (Seoul, Korea). U87 cells were grown in Dulbeccoe Korea Cell Line Bank (Seoul, Korea). DU145 and MCF-7 cells were grown in RPMI 1640 culture medium (Welgene). DMEM and RPMI 1640 were supplemented with 10\% fetal bovine serum (HyClone, Logan, UT), $100 \mathrm{U} / \mathrm{mL}$ penicillin, and $100 \mathrm{mg} / \mathrm{mL}$ streptomycin (GIBCO, Grand Island, NY, USA). Cancer cells were cultured as previously described [12]. Cells were seeded into 96-well cell culture plates and allowed to grow for $24 \mathrm{~h}$ prior to treatment with oleanolic acid (Sigma, St. Louis, MO, USA).

\subsection{Cytotoxicity assays}

The effects of oleanolic acid on tumor cell proliferation were evaluated using the 3-(4,5 dimethylthiazol-2-yl)-2,5-diphenyltetrazolium bromide (MTT; Genetrone, Seoul, Korea) analysis.

\subsection{Measurement of apoptosis}

Apoptotic cells were quantified using Annexin V-FITC/PI double staining, with an Annexin V-FITC apoptosis detection kit (BD Pharmingen, San Diego, CA, USA) according to the manufacturer's instructions. 


\subsection{Cell cycle analysis}

Cells $\left(5 \times 10^{4}\right.$ cells/well in 6-well plates) were treated with OA for $24 \mathrm{~h}$. After incubated, cells were harvested and washed once with ice cold phosphate-buffered saline (PBS), and fixed with ice-cold $70 \%$ ethanol at $4{ }^{\circ} \mathrm{C}$. After removing the remaining PBS, cell pellets were suspended in 300 $\mu \mathrm{L}$ propidium iodide (PI) solution $\left(69 \mu \mathrm{M}\right.$ PI in $38 \mathrm{mM}$ sodium citrate) and incubated at $37^{\circ} \mathrm{C}$ for 45 min in the dark. Cells were then analyzed using a flow cytometer (Becton Dickinson, CA, USA). The percentage of cell distribution was calculated using Cell Quest software. Specific inhibitors of PD98059 (PD) were purchased from Cell Signaling Technology (Beverly, MA, USA) and Calbiochem (San Diego, CA, USA).

\subsection{Western blot analysis}

Cells were lysed in ice-cold RIPA lysis buffer and $1 \mathrm{mM}$ phenylmethylsulfonyl fluoride (protease inhibitor). The membranes were then incubated with primary antibodies including p53, p-AKT (Epitomics, Burlingame, CA, USA), cytochrome c, Bax, caspase-3, p-ERK, p-JNK, CDK2, p27, and $\beta$-actin (Cell Signaling Technology), p21, cyclin B1 (Santa Cruz Biotechnology, Santa Cruz, CA, USA) antibodies. Membranes were then incubated with a goat anti-rabbit IgG $(\mathrm{H}+\mathrm{L}) \mathrm{HRP}$-conjugated secondary antibody (Zymax, San Francisco, CA, USA). The antigen-antibody complexes were visualized by enhanced chemiluminescence. Densitometric analysis of the signal was performed using a C-DiGit Blot Scanner (Li-COR Inc., Lincoln, NE, USA). Relative expression was quantified using Image J (NIH, Bethesda, Rockville, MD, USA) and compared to $\beta$-actin.

\subsection{Statistical analyses}

The statistical analysis was performed using SPSS 18.0 (SPSS Inc., Chicago, IL, USA). Averages and standard deviations were calculated and differences between groups were assessed using the analysis of variance method and Duncan's multiple range test. A difference was considered significant if $\mathrm{p}<0.05$.

\section{Results}

\subsection{Cytotoxic activity of $O A$ in cancer cells}

To investigate the effects of OA on cell viability in cancer cells (DU145, MCF-7, and U87 cell lines), we treated the cells with $0,25,50$, and $100 \mu \mathrm{g} / \mathrm{mL}$ OA for $24 \mathrm{~h}$ and assessed cell proliferation using the MTT assay (Table 1). The IC 50 values, following OA treatment, were 112.56, 132.29, and $163.60 \mu \mathrm{g} / \mathrm{mL}$ in the DU145, MCF-7, and U87 cell lines, respectively. OA presented an anti-tumor effect in cancer cells, we investigated the underlying molecular mechanism.

Table 1. Cytotoxicity of Oleanolic acid (OA) in cancer cells (DU145, HeLa, U87, and MCF-7) as determined using the MTT assay.

\begin{tabular}{cccc}
\hline & DU145 & MCF-7 & U87 \\
\hline $\mathrm{IC}_{50}(\mu \mathrm{g} / \mathrm{mL})$ & 112.57 & 132.29 & 163.60 \\
\hline
\end{tabular}

\subsection{Proapoptotic activity of $O A$ in cancer cells}

To determine whether the anti-cancer effects of OA were associated with apoptosis, we quantified apoptosis in OA-treated DU145, MCF-7, and U87 cells were treated with OA (0, 50, and $100 \mu \mathrm{g} / \mathrm{mL}$ ) for $24 \mathrm{~h}$, and apoptotic cells were quantified by Annexin V-FITC staining (Figure 1(a)). OA increased the number of late-apoptotic cells according to dose-dependent manner. As shown in 
Figure $1(\mathbf{b})$, treatment with 50 and $100 \mu \mathrm{g} / \mathrm{mL} \mathrm{OA}$ resulted in $7.69 \%$ and $27.0 \%$ apoptotic cells in DU145, 20.9\% and $27.0 \%$ apoptotic cells in MCF-7, and $4.02 \%$ and $15.7 \%$ apoptotic cells in U87 respectively, as compared with $0 \%$ (early-apoptotic and late-apoptotic cells) in the control. Our results show that OA treatment decreased cell proliferation and increased apoptosis in these cancer cell lines.

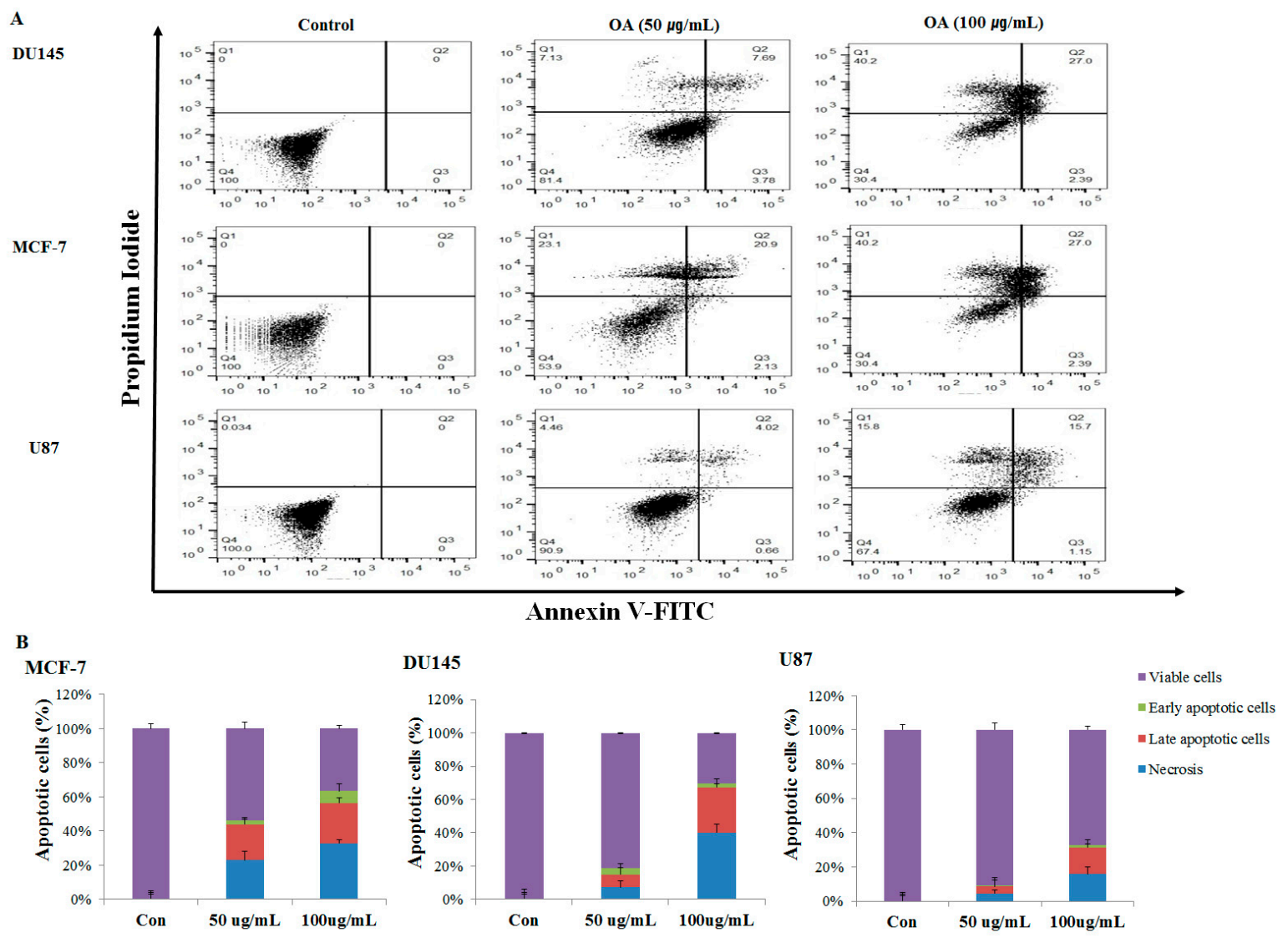

Figure 1. Dose dependence of oleanolic acid (OA)-induced apoptotic cell death in DU145, MCF-7, and U87 cells. (a) Annexin V-FITC/PI staining for the detection of apoptotic cells. After treatment with OA $(0,50$, or $100 \mu \mathrm{g} / \mathrm{mL})$, cells were stained with Annexin V-FITC/PI and subjected to flow cytometry. (b) Quantitation of the FACS data shown in (a). Results are expressed as the means \pm SD.

\subsection{Effects of OA treatment on the expression of apoptosis-related proteins}

To characterize the molecular mechanism of OA-induced apoptosis in DU145, MCF-7, and U87 cells, we measured the expression of apoptosis-related proteins (p53, cytochrome c, Bax, caspase-3, PARP-1) by western blot (Figure 2). OA treatment significantly increased the expression of apoptosis-related proteins in DU145, MCF-7, and U87 cells. The expression of p53 and cytochrome c in DU145 cells treated with $100 \mu \mathrm{g} / \mathrm{mL}$ OA was significantly higher (10.11, 74.70-fold) than that in the control group. Moreover, the expression of Bax and caspase-3 increased 1.51 and 3.16-fold in cells treated with $100 \mu \mathrm{g} / \mathrm{mL}$ OA, compared to that in the control group. In addition, the expression of p53 and cytochrome c in MCF-7 cells treated with $100 \mu \mathrm{g} / \mathrm{mL}$ OA increased, and the expression of Bax and PARP-1 increased 1.79 and 2.16-fold, compared to that in the control group. Similarly, an upregulation of p53 and cytochrome c was observed in U87 cells treated with OA. These results show that apoptosis is induced in cancer cell lines treated with OA. 


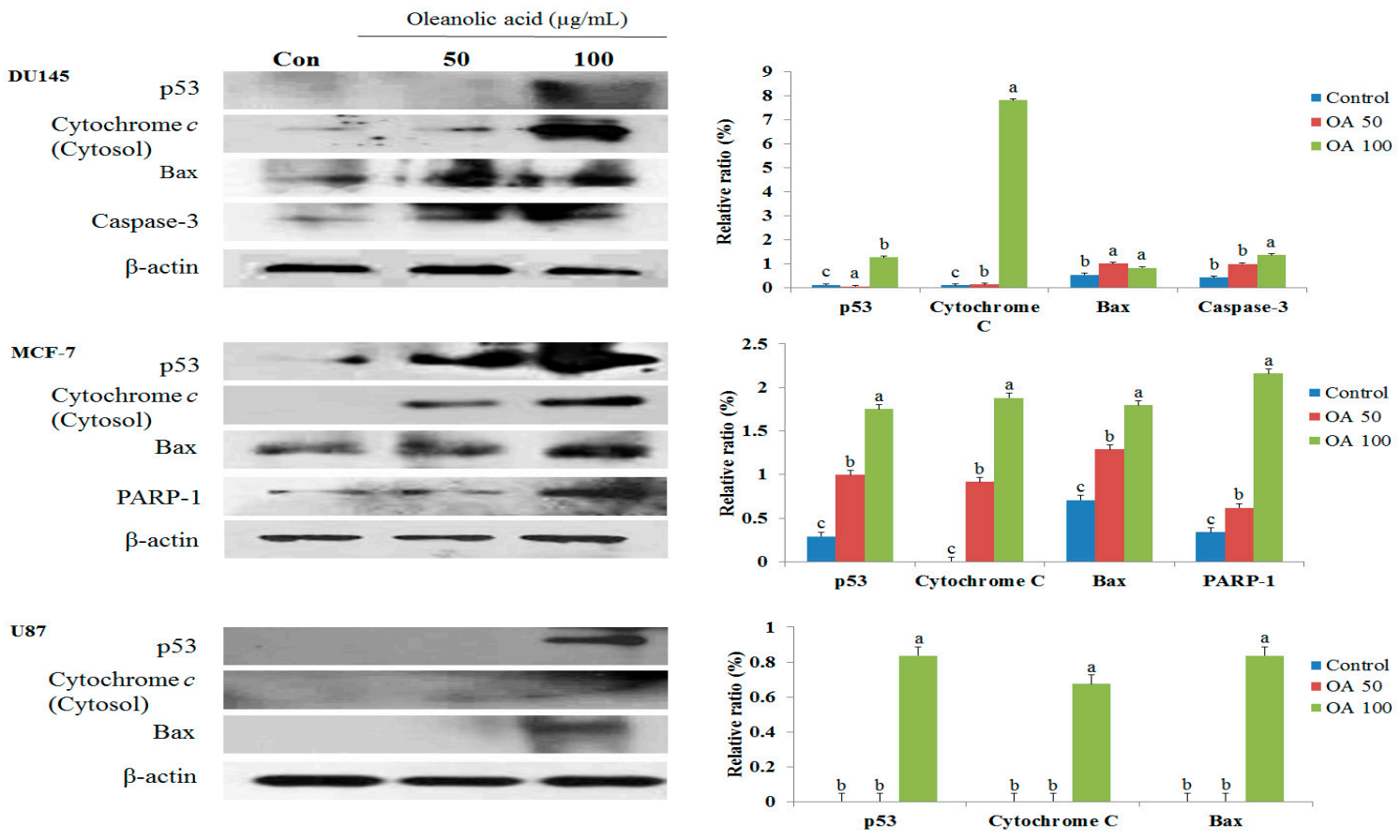

Figure 2. Effect of oleanolic acid (OA) on apoptosis in DU145, MCF-7 and U87 cells. The cells were treated with 50 and $100 \mu \mathrm{g} / \mathrm{mL}$ OA for $24 \mathrm{~h}$. After treatment, the expression of p53, cytochrome c, Bax, PARP-1, and caspase-3 was determined by western blot. Equal loading was confirmed by $\beta$-actin quantification. The relative density of the proteins was measured and normalized to $\beta$-actin (arbitrarily set at 1$)$. Results are expressed as means \pm SD. Significant differences $(p<0.05)$ are represented using different letters.

\subsection{Effects of $O A$ on cell cycle progression}

To investigate the effects of OA on cell cycle progression, we treated DU145, MCF-7, and U87 cells with OA (at 0, 50, and 100 OA-treated DU145, MCF- and analyzed the different stages of the cell cycle using flow cytometry. As shown in Figure 3(b), OA-treated DU145 cells showed enhancement in the percentage of cells in G2, compared to that in control cells $(P<0.05)$. Treatment with 50 and $100 \mu \mathrm{g} / \mathrm{mL}$ OA resulted in $23.15 \%$ and $27.62 \%$ of cells arrested in the G2 phase, respectively. Conversely, MCF-7 cells treated with growth-suppressive concentrations of OA $(0,50$, and 100 $\mu \mathrm{g} / \mathrm{mL}$ concentrations) decrease in the percentage population (64.62 and $67.21 \%$, respectively), and a decrease in the S and G2 populations, compared to those in the control. U87 cells showed no significant differences. These observations suggest that OA implicated cell cycle progression in a cell type-dependent manner. 
A

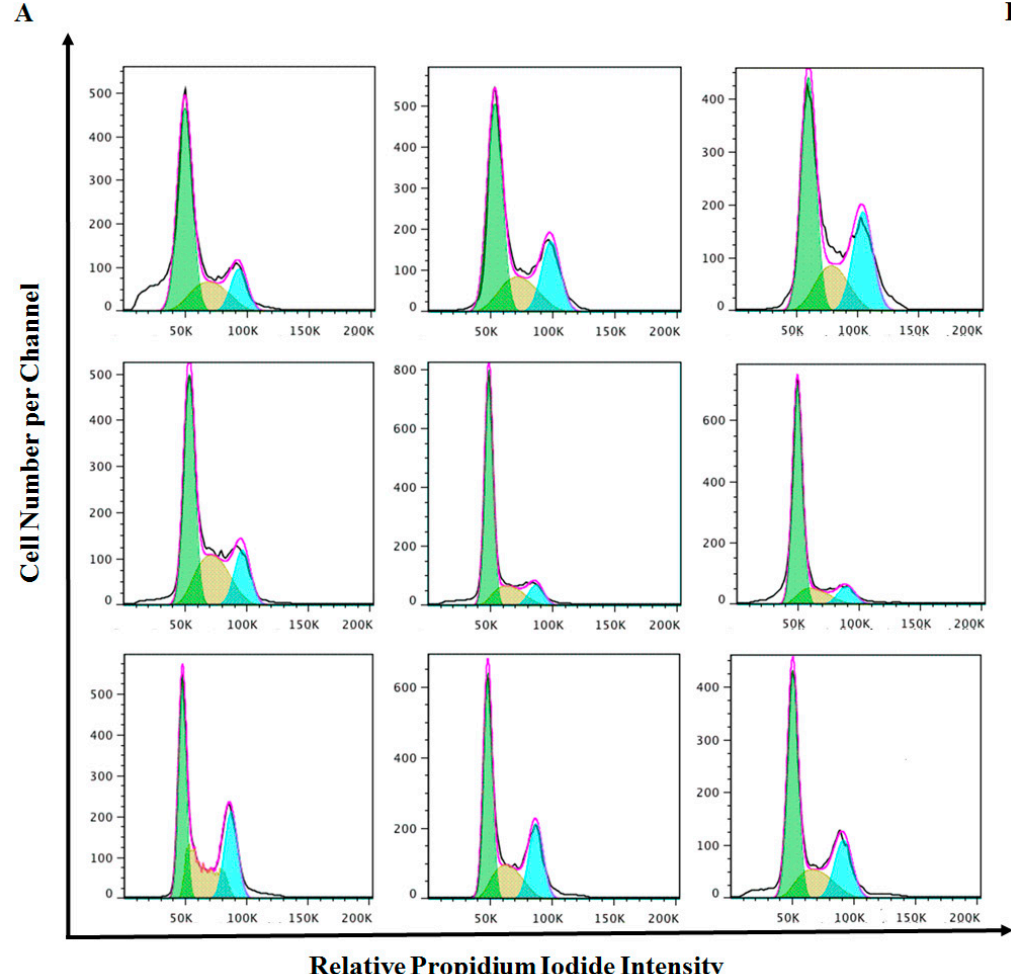

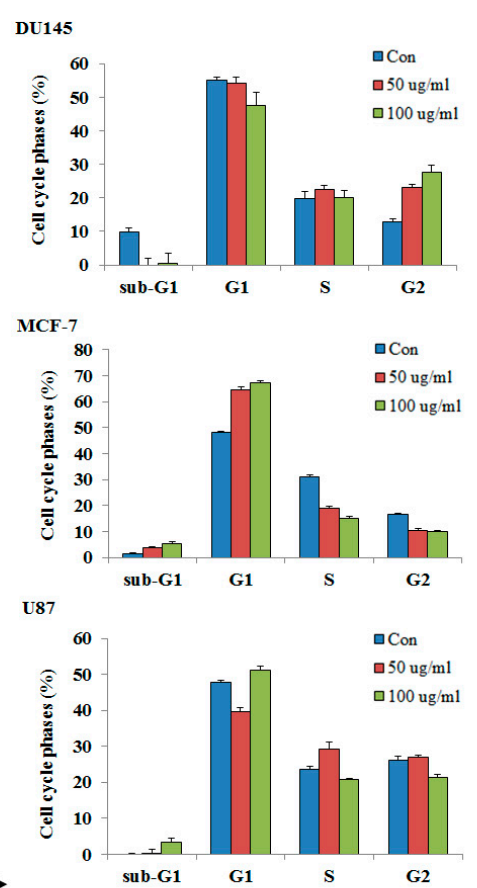

Figure 3. Effect of oleanolic acid (OA) treatment on cell cycle progression in DU145, MCF-7, and U87 cells. (A) Flow cytometric analysis of cell cycle distribution in cells treated with 0, 50, and 100 $\mu \mathrm{g} / \mathrm{mL}$ OA. (B) Histograms showing the percentages of cells in the G1, S, and G2 phases of the cell cycle. Results are expressed as the means \pm SD.

\subsection{Effects of $O A$ on the expression of cell cycle regulators}

As shown by western blot analysis in Figure 4, OA caused the arrest of cells in the G2 phase in DU145 cells, and the G1 phase in MCF-7 cells. We next assessed its effects on the G1 and G2 cell cycle regulators, including cyclin B1, p21, cyclin E, CDK 2, and p27. Treatment with $100 \mu \mathrm{g} / \mathrm{mL}$ OA in DU145 cells showed that expression of cyclin B1 and cyclin E considerably decreased compared to that in the control, while the level of p21 (13.64-fold) and CDK2 (1.46-fold) increased under the same treatment. In MCF-7 cells, treatment with $100 \mu \mathrm{g} / \mathrm{mL}$ OA decreased the expression of cyclin B1, cyclin E, and CDK2 by 1.03-, 0.04-, and 12.02-fold with respect to that of the control group, respectively, while the expression of p21 and p27 increased 9.09- and 4.78-fold, respectively. Treatment of U87 cells with $100 \mu \mathrm{g} / \mathrm{mL}$ OA significantly increased the expression of cyclin B1, p21, and p27, and decreased the level of CDK2. 


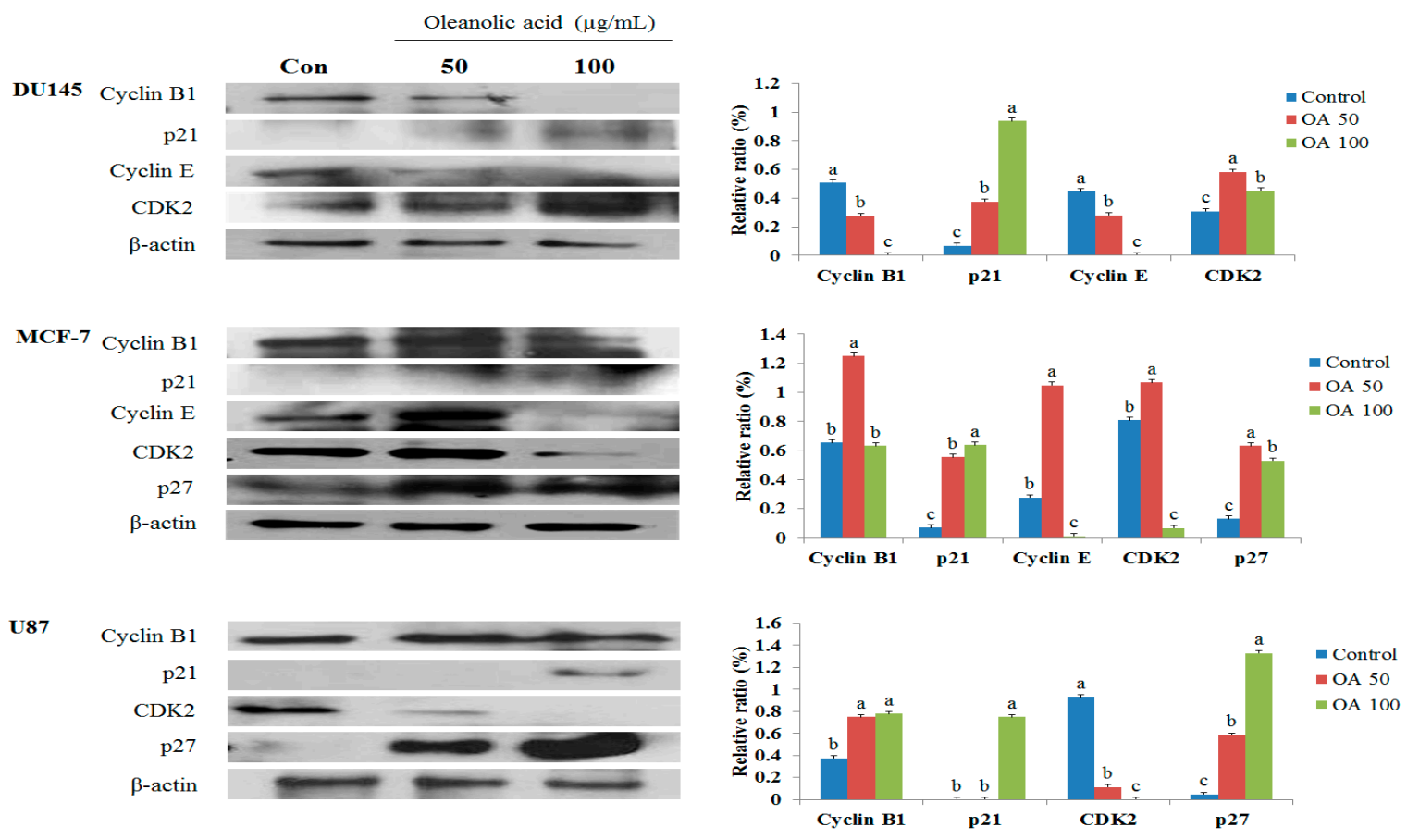

Figure 4. Effect of oleanolic acid (OA) treatment on the levels of cell cycle regulatory proteins in DU145, MCF-7, and U87 cells. The cells were treated with 50 and $100 \mu \mathrm{g} / \mathrm{mL}$ OA for $24 \mathrm{~h}$. After treatment, the expression of cyclin B1, p21, cyclin E, CDK2, and p27 was determined by western blot analysis. Equal loading was confirmed by $\beta$-actin quantification. For each protein, the relative density was measured and normalized to the $\beta$-actin bands (arbitrarily set at 1 ). The results are expressed as the means \pm SD. Significant differences $(\mathrm{p}<0.05)$ are represented using different letters.

\subsection{Effects of $O A$ on ERK, JNK, and AKT activation in cancer cells}

To determine whether OA affects MAPK activation, we measured ERK, JNK and AKT activation in response to OA in DU145, MCF-7, and U87 cells. As shown in Figure 5, the phosphorylation levels of JNK and AKT increased after OA treatment in DU145 cells. However, the phosphorylation levels of ERK decreased. In MCF-7 cells, the levels of p-AKT significantly decreased, while those of $\mathrm{p}$-ERK and p-JNK increased. Moreover, we measured the activation of p-ERK, p-AKT and p-JNK in U87 cells. An increase in p-ERK, p-AKT, and p-JNK was observed following the $100 \mu \mathrm{g} / \mathrm{mL}$ OA treatment. Our results demonstrate that the expression of proteins involved in MAPK kinase signaling was differently regulated in various cell lines. 


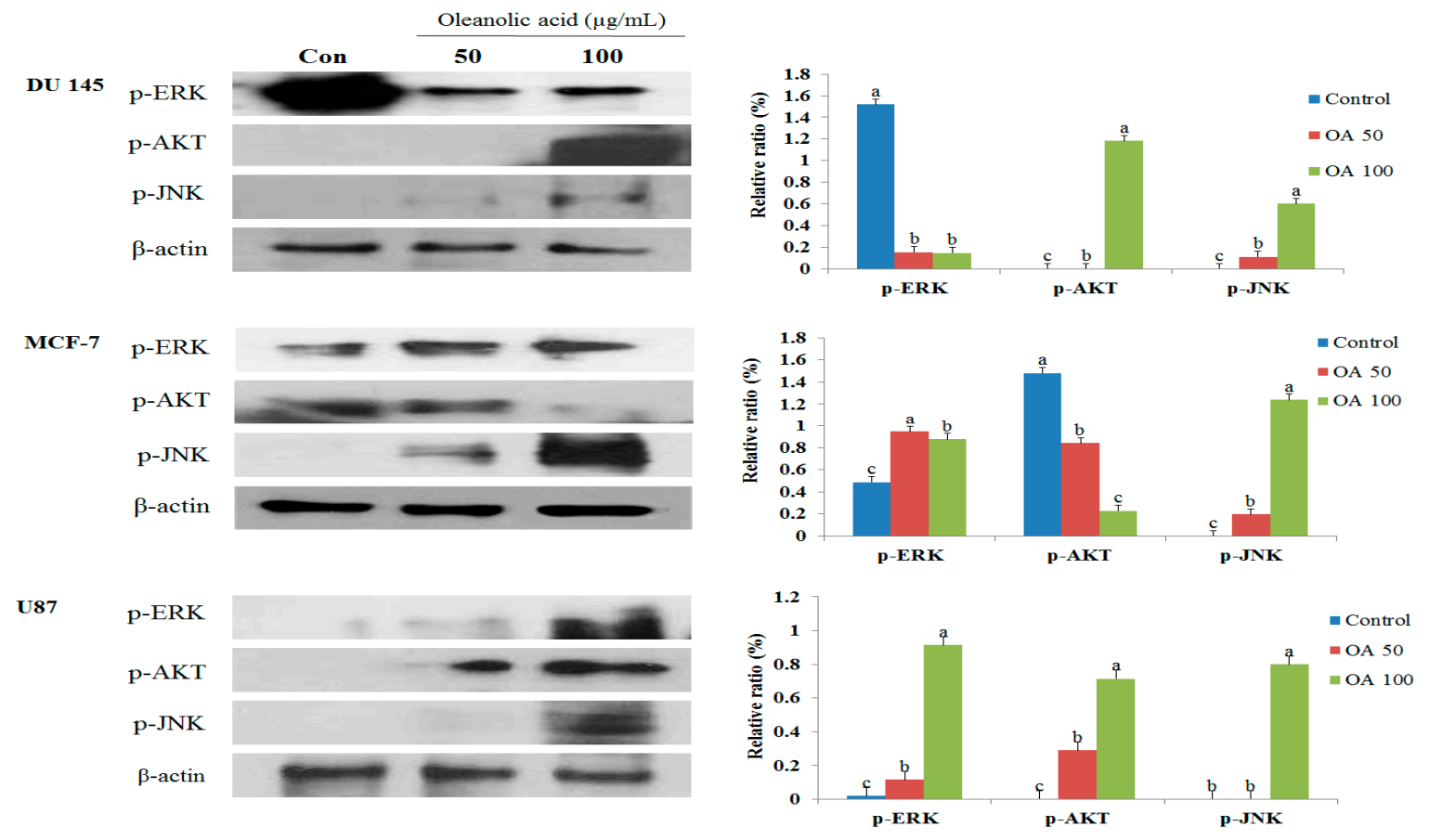

Figure 5. Effect of oleanolic acid (OA) on MAPK kinase signaling in DU145, MCF-7, and U87 cells. The cells were treated with 50 and $100 \mu \mathrm{g} / \mathrm{mL}$ OA for $24 \mathrm{~h}$. After treatment, the expression of p-ERK, p-AKT, and p-JNK was determined using western blot analysis. Equal loading of proteins was confirmed using $\beta$-actin quantification. Results are expressed as the means \pm SD. Significant differences $(\mathrm{p}<0.05)$ are represented using different letters.

\subsection{The inhibition of the MAPK signaling pathway is involved in the OA-induced anticancer effect}

To further test whether the inhibition of the ERK signaling pathway is involved in the OA-induced anticancer activities in DU145, MCF-7, and U87 cell lines, the cells were treated with PD98059, a selective inhibitor of MAPK that disrupts the downstream expression of p-ERK, p-AKT, and p-JNK. As shown in Figure 6, treatment with PD98059 increased the activation of p-ERK in DU145 cells, compared to that observed with OA alone. Moreover, treatment with PD98059 alone showed that p-AKT and p-JNK levels decreased in DU145 cells. Combination treatment with PD98059 and OA further decreased the p-ERK and p-JNK levels in DU145 cells, compared with those observed after treatment with PD98059 alone. In DU145 cells treated with a combination of PD98059 and OA, p-JNK levels did not change compared to its levels following treatment with PD98059 alone. We found that treatment with PD98059 in MCF-7 cells reduced the expression of p-ERK and p-JNK, compared to that detected with OA alone. However, in MCF-7 treated with a combination of PD98059 and OA, no changes were observed in p-ERK, p-AKT, and p-JNK levels, compared to their levels in cells treated with PD98059 alone. In U87 cells treated with PD98059, p-ERK and p-JNK expression decreased, compared with that in cells treated with PD98059 alone. However, the expression of p-AKT increased in U87 cells treated with PD98059. The expression of p-ERK, p-AKT, and p-JNK in U87 cells treated with a combination of PD98059 and OA was lower than that observed in cells treated with PD98059 alone. 


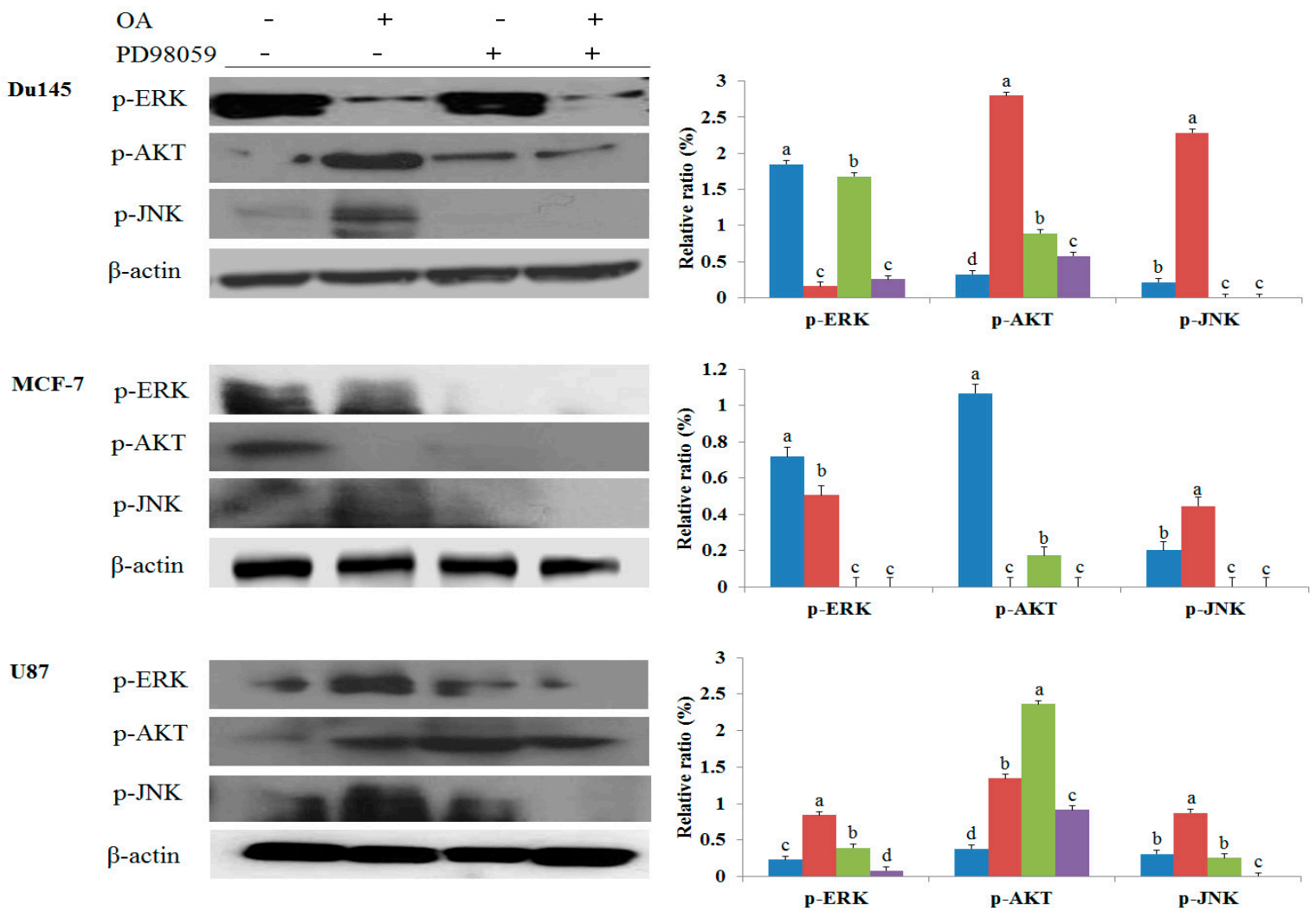

Figure 6. Effect of oleanolic acid (OA) or PD98059, alone or in combination, on the expression of proteins involved in MAPK kinase signaling in DU145, MCF-7, and U87 cells. The cells were treated with OA $(100 \mu \mathrm{g} / \mathrm{mL})$ and PD98059 $(50 \mu \mathrm{M})$, alone or in combination, for $24 \mathrm{~h}$, and the expression of p-ERK, p-AKT, and p-JNK was determined by western blot. Equal loading of proteins was confirmed using $\beta$-actin quantification. Results are expressed as the means \pm SD. Significant differences $(p<0.05)$ are represented using different letters.

\subsection{PD98059 enhanced the OA-induced apoptosis in U87 cells}

To determine whether the anti-cancer effects of OA were associated with apoptosis, we quantified apoptosis in DU145, MCF-7, and U87 cells treated with a combination of PD98059 and OA, using Annexin V-FITC staining. As shown in Figure 7, treatment with PD98059 in DU145 cells did not affect apoptosis, when compared to the control. However, in PD98059 and OA combination-treated DU145 cells, the number of late-apoptotic cells increased compared to that in cells treated with PD98059 alone. Moreover, the number of late apoptotic cells in MCF-7 cells treated with PD98059 alone was higher than that in control. In PD98059 and OA combination-treated MCF-7 cells, the number of apoptotic cells was lower than that in cells treated with PD98059 alone. U87 cells treated with PD98059 displayed a decrease in late-apoptotic cells, compared to those treated with OA alone. However, in U87 cells treated with a combination of PD98059 and OA, the number of late-apoptotic cells increased compared with that in cells treated with OA. Our results show that pretreatment with the PD98059 MAPK inhibitor significantly increased the OA-induced apoptosis against U87 cells, but did not affect cell death in DU145 and MCF-7 cells. 
A

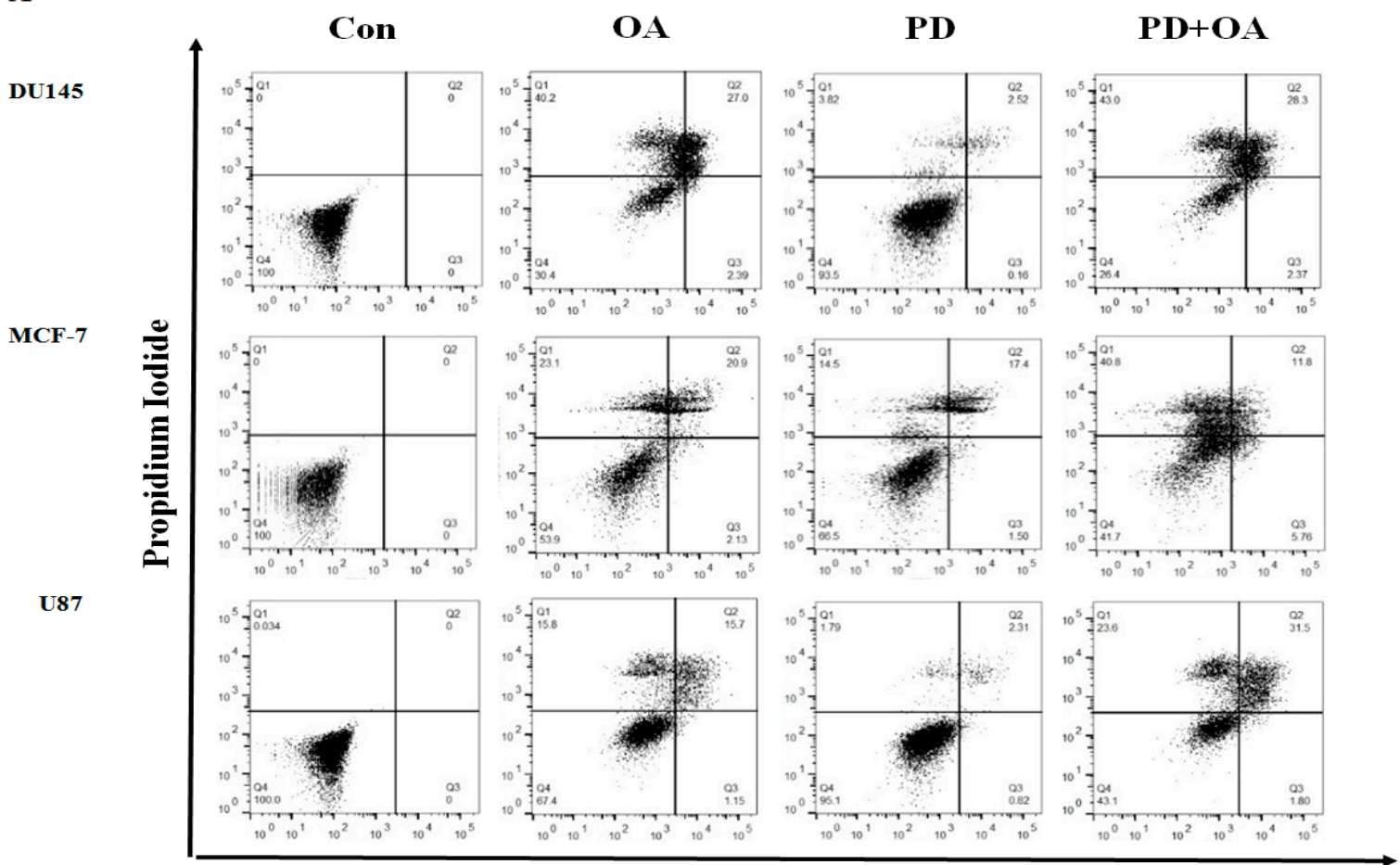

Annexin V-FITC

B

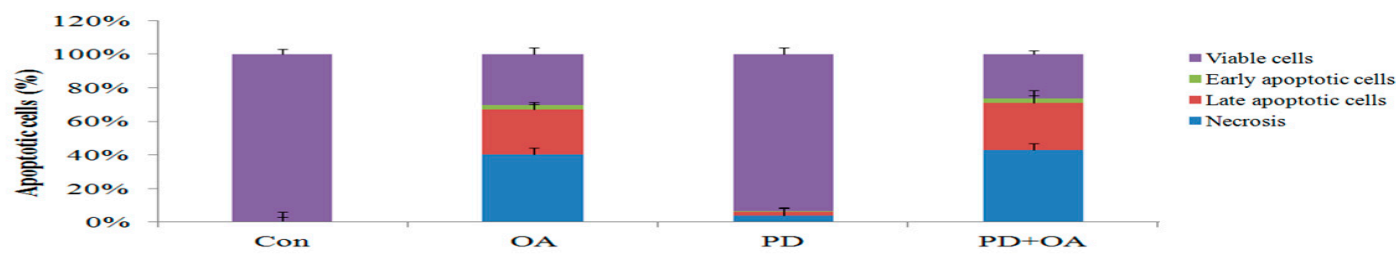

MCF7

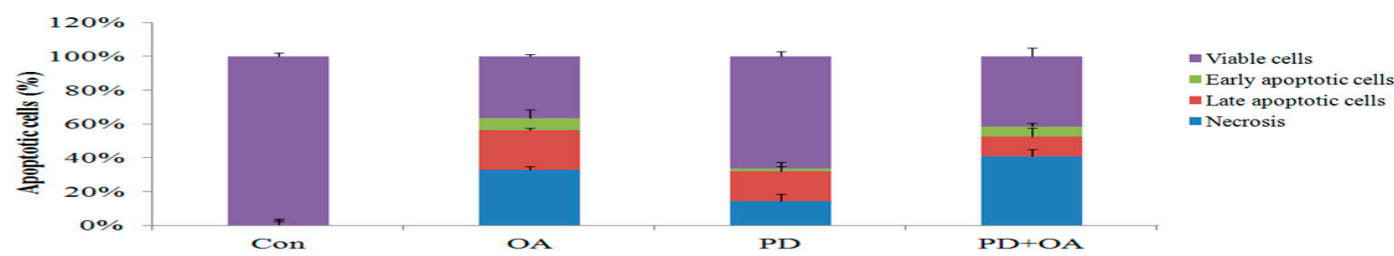

U87

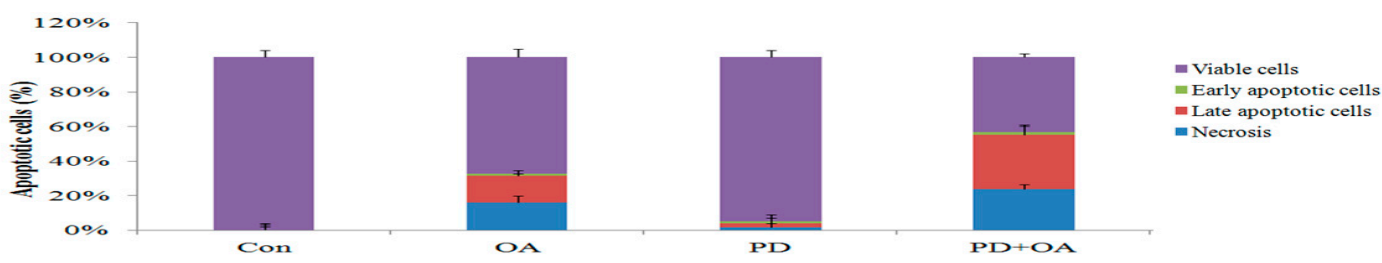

Figure 7. Apoptotic cell death in DU145, MCF-7, and U87 cells treated with oleanolic acid (OA) or PD98059, alone or in combination. (a) Annexin V-FITC/PI staining for the detection of apoptotic cells. After treatment with OA $(100 \mu \mathrm{g} / \mathrm{mL})$ or PD98059 $(50 \mu \mathrm{M})$, alone or in combination, cells were stained with Annexin V-FITC/PI and subjected to flow cytometry. (b) Quantitation of the FACS data shown in (a). Results are expressed as the means \pm SD.

3.9 The OA-induced G1, S, and G2 phase arrest is due to the inhibition of the MAPK signaling pathway

To investigate whether the inhibition of the ERK signaling pathway alters the distribution of cells in distinct phases of the cell cycle, we treated DU145, MCF-7, and U87 cells with PD98059 alone, or with a combination of PD98059 and OA, and analyzed them using flow cytometry. As shown in 
Figure 8, treatment with PD98059 increased the percentage of cells in the G1 phase, as compared to that in DU145 cells treated with OA alone. Moreover, in DU145 cells treated with a combination of PD98059 and OA the number of cells in the G1 phase increased compared with that in cells treated with PD98059 alone. A higher percentage of cells arrested in the S phase was found following treatment with PD98059 alone, or with a combination of PD98059 and OA in the MCF-7 cell line. We also noted that U87 cells treated with PD98059 alone showed a significant increase in the fraction of cells in the G2 phase of the cell cycle, while the PD98059 and OA combination treatment enhanced the percentage of cells in the S phase compared with that in cells treated with PD98059 alone.
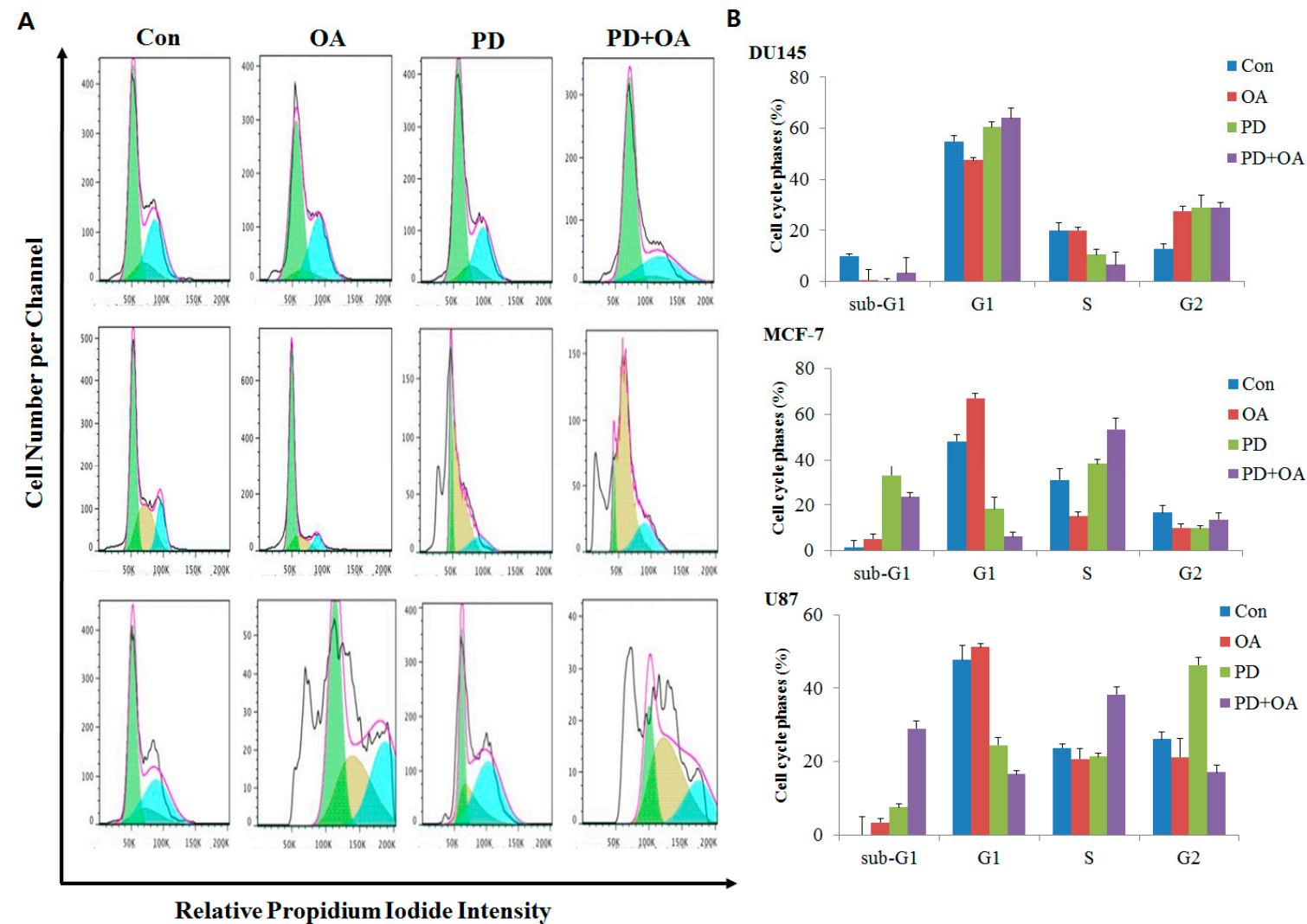

Figure 8. Effect of oleanolic acid (OA) or PD98059, alone or in combination, on the cell cycle distribution of DU145, MCF-7, and U87 cells. The cells were treated with OA $(100 \mu \mathrm{g} / \mathrm{mL})$ and PD98059 $(50 \mu \mathrm{M})$, alone or in combination, for $24 \mathrm{~h}$, and the cell cycle distribution was estimated using flow cytometry, as described in the methods. (a) Flow cytometry analysis of the cell cycle distribution in cells treated with OA $(100 \mu \mathrm{g} / \mathrm{mL})$ or PD98059 $(50 \mu \mathrm{M})$, alone or in combination (b) Histograms showing the percentages of cells in the G1, S, and G2 phases of the cell cycle. Results are expressed as the means \pm SD.

\section{Discussion}

Our study investigated the effect of oleanolic acid on the viability, apoptosis, and cell cycle arrest of DU145 prostate cancer cells, MCF-7 breast cancer cells, and U87 human glioblastoma cells. We investigated that treatment with OA inhibited cell proliferation in all of these cell lines. The IC 50 values for the cytotoxic effect of OA were 112.56, 132.29, and $163.60 \mu \mathrm{g} / \mathrm{mL}$ in the DU145, MCF-7, and U87 cell lines, respectively. Our results suggest that OA treatment affects cell-cycle progression and apoptosis in a cell type dependent manner.

Apoptosis is regulated through endonucleases and the sequential activation of cascade of caspases enzymes in the cancer cell. These enzymes carry out a series of studies guiding to the characteristic changes related with apoptosis, including cell shrinkage, chromatin condensation, 
fragmentation and plasma-membrane blebbing [16]. Bax controls cell death through mitochondrial disruption, which lead to release of cytochrome $\mathrm{c}$ into the cytosol [18]. Caspases, and particularly caspase-3, play important roles in executing apoptosis, and an increase in the activity of caspases is observed in cells undergoing apoptosis $[19,20]$. The tumor suppressor gene p53 can be activated under a variety of conditions, including hypoxia, heat shock and DNA damage [16]. p53 regulates the transcription of genes associated with cell cycle arrest (e.g., Gadd45 and p21) and apoptosis (e.g., DR5, Bax, caspase-3, Apaf-1, Fas, the p53-inducible gene, and Noxa) [16]. As shown in Figure $1,27.0 \%, 27.0 \%$, and $15.7 \%$ of apoptotic cells were detected in the DU145, MCF-7, and U87 cell lines respectively. Moreover, OA treatment decreased cell viability and increased apoptosis in a prostate cancer cell line. Furthermore, our results show that the expression levels of the apoptosis-associated p53, cytochrome c, Bax, and caspase-3 proteins were significantly increased in cancer cells treated with OA. Our results are consistent with a previously report, showing that dextrose-OA induces apoptosis in human osteosarcoma cell lines (HOS, U2-OS and MG-63), and in the LM-8 mouse osteosarcoma cell line [17].

Cell cycle analysis result demonstrated that $\mathrm{OA}$ treatment increased the percentage of cells in the G2 phase in DU145 cells. However, MCF-7 cells treated with growth-suppressive concentrations of OA, displayed increases in the G1 population. The protein level of p-JNK increased, but that of cyclin B1 decreased after treatment with OA in DU145 cells. In addition, in MCF-7 cells, the expression levels of $\mathrm{p}-\mathrm{AKT}$, cyclin $\mathrm{E}$, and CDK2 reduced in a dose-dependent manner, while the levels of p-ERK and p-JNK increased. Our results demonstrate that the JNK signaling pathway modulates the mitochondrial pathway and causes the G2 phase arrest in DU145 cells treated with OA. Furthermore, our study suggests that the MAPK signaling pathway is required for the regulation of the p53-mediated G1 phase cell cycle arrest in MCF-7 cells. Interestingly, the effect of the OA treatment in our study differed from the one noted in other reports. For example, in HL-60 myeloid leukemia cell line and sHCT15 colon carcinoma, OA treatment suppressed that of cells in S phase and enhanced the percentage of cells in the G0/G1 phase [22,21]. Gambogic acid from Garcinia hanburyi has been reported to induce G2 arrest in BGC-823 human gastric carcinoma cells, similar to the effects we observed with OA [14]. Our observations suggest that OA related with the cell cycle progression in a cell type-dependent manner.

In conclusion, treatment with OA inhibited the growth of DU145, MCF-7, and U87 cancer cell lines. Moreover, OA treatment increased the expression of the p53, cytochrome c, Bax, and caspase-3 apoptosis-promoting proteins. Our cell cycle analysis showed that OA treatment resulted in an accumulation of G2 phase cells, with a concomitant decrease in G1 phase cells in the DU145 cell line. However, OA treatment in the MCF-7 and U87 cell lines resulted in an accumulation of cells in the G1 phase, and in a concomitant decrease in cells in the G2 phase. OA led to an increase in p-ERK and p21/WAF-1 expression, and a decrease in cyclin B1 protein levels in DU145 cells. In MCF-7 cells, the expression levels of p21 and p53 increased, whereas those of cyclin E, CDK2, and p-AKT decreased in a dose-dependent manner. Our studies showed that the anti-cancer effects of OA on cancer cells are orchestrated by the activation of apoptosis and the reduction of cell cycle progression. These findings raise the possibility that OA might be effective for the treatment of prostate and breast cancers, as well as for the treatment of cancers derived from other tissue types.

Acknowledgments: This paper was supported by Konkuk University.

Author Contributions: G-J K and H-J J conducted the experiments and analyzed the data. K-H C, $\mathrm{K}-\mathrm{J} \mathrm{L}$, and $\mathrm{J} \mathrm{H}$ A designed the experiments.

Conflicts of Interest: The authors declare no conflict of interest.

\section{References}

1. Kanavos, P. The rising burden of cancer in the developing world. Ann. Oncol. 2006, 17, viii15-viii23.

2. Bray, F.; Jemal, A.; Grey, N.; Ferlay, J.; Forman, D. Global cancer transitions according to the 
human development index (2008-2030): a population-based study. Lancet Oncol. 2012, 13, 790-801.

3. National cancer information center. http://www.cancer.go.kr/mbs/cancer/

4. Reddy, V.G.; Khanna, N.; Singh, N.; Vitamin C augments chemotherapeutic response of cervical carcinoma HeLa cells by stabilizing p53. Biochem Bioph Res Co. 2001, 282, 409-415.

5. Ghate N.B.; Chaudhuri $_{\llcorner}$D.; Sarkar R.; Sajem $_{\llcorner}$A.L.; Panja S.; Rout $_{\llcorner}$J.; Mandal N1. An Antioxidant extract of tropical Lichen, parmotrema reticulatum, induces cell cycle arrest and apoptosis in breast carcinoma cell line MCF-7. PLoS One. 2013, 8, e82293

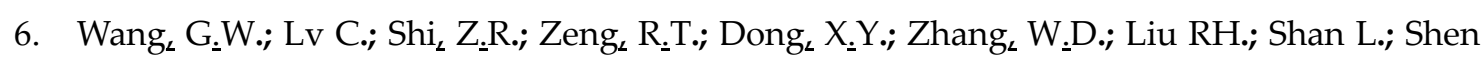
YH. Abieslactone induces cell cycle arrest and apoptosis in human hepatocellular carcinomas through the mitochondrial pathway and the generation of reactive oxygen species. PLoS One. 2014, 9, e115151

7. Zhu, X.; Wang, K.; Zhang, K.; Zhu, L.; Zhou, F.; Ziyuglycoside II induces cell cycle arrest and apoptosis through activation of ROS/JNK pathway in human breast cancer cells. Toxicol.Lett. 2014, 227, 65-73

8. Kim, G.J.; Song, D.H.; Yoo, H.S.; Chung, K.H.; Lee, K.J.; An, J.H. Hederagenin supplementation alleviates the pro-inflammatory and apoptotic response to alcohol in rats. Nutrients. 2017, 9,41

9. Walensky, L.D.; Gavathiotis, E. BAX unleashed: the biochemical transformation of an inactive cytosolic monomer into a toxic mitochondrial pore. Trends Biochem Sci. 2011, 36, 642-652.

10. Czabotar, P.E.; Colman, P.M.; Huang, D.C. Bax activation by Bim? Cell Death Differ. 2009, 16, 1187-1191.

11. Dejean, L.M.; Martinez-Caballero, S.; Guo, L.; Hughes, C.; Teijido, O.; Ducret, T.; Ichas, F.; Korsmeyer, S.J.; Antonsson, B.; Jonas, E.A.; Kinnally, K.W. Oligomeric Bax is a component of the putative cytochrome $c$ release channel MAC, mitochondrial apoptosis-induced channel. Mol. Biol. Cell. 2005, 16, 2424-2432.

12. Liu, C.; Zhu, Y.; Lou, W. Nadiminty N, Chen X, Zhou Q, Shi XB, deVere White RW, Gao AC. Functional p53 determines docetaxel sensitivity in prostate cancer cells. Prostate. 2013, 73, 418-427.

13. Singh, S.K.; Banerjee, S.; Acosta, E.P.; Lillard, J.W.; Singh, R. Resveratrol induces cell cycle arrest and apoptosis with docetaxel in prostate cancer cells via a p53/ p21WAF1/CIP1 and p27KIP1 pathway. Oncotarget. 2017, 8, 17216-17228

14. Sa, G.; Das, T. Anti cancer effects of curcumin: cycle of life and death. Cell Div. 2008, 3,14.

15. Suzuki, T.; Urano, T.; Miki, Y.; Moriya, T.; Akahira, J.; Ishida, T.; Horie, K.; Inoue, S.; Sasano, H. Nuclear cyclin B1 in human breast carcinoma as a potent prognostic factor. Cancer Sci. 2007, 98, 644-651.

16. Kawamoto, H.; Koizumi, H.; Uchikoshi, T. Expression of the G2-M checkpoint regulators cyclin B1 and cdc2 in nonmalignant and malignant human breast lesions: immunocytochemical and quantitative image analyses. Am. J. Pathol. 1997, 150, 15-23.

17. Oguro, T.; Liu, J.; Klaassen, C.D.; Yoshida, T. Inhibitory Effect of Oleanolic acid on 12-O-tetradecanoylphorbol-13-acetate-lnduced gene expression in mouse skin. Toxicol. Sci. 1998, 45, 88-93. 
18. Perez-Camino, M.C.; Cert, A. Quantitative determination of hydroxy pentacyclic triterpene acids in vegetable oils. J. Agr. Food Chem. 1999, 47, 1558-1562.

19. Liu, J. Pharmacology of oleanolic acid and ursolic acid. J. Ethnopharmacol. 1995, 49, 57-68.

20. Akihisa, T.; Kamo, S.; Uchiyama, T.; Akazawa, H.; Banno, N.; Taguchi Y.; Yasukawa K. Cytotoxic activity of Perilla frutescens var. japonica leaf extract is due to high concentractions of oleanolic and ursolic acids. J. Nat. Med. 2006, 60, 331-333.

21. Li, H.F.; Wang, X.A.; Xiang, S.S.; Hu, Y.P.; Jiang, L.; Shu, Y.J.; Li, M.L.; Wu, X.S.; Zhang, F.; Ye, Y.Y.; Weng, H.; Bao,RF.;Cao,Y.; Lu,W.; Dong,Q.; Liu,YB.; Oleanolic acid induces mitochondrial-dependent apoptosis and G0/G1 phase arrest in gallbladder cancer cells. Drug Des. Devel. Ther. 2015, 9, 3017-30.

22. Chiang, Y.M.; Chang, J.Y.; Kuo, C.C.; Chang, C.Y.; Kuo, YH. Triterpenes from aerial roots of Ficus microcarpa. Phytochemistry. 2005, 64, 495-501.

23. Ng, Y.P.; Chen, Y.; Hu, Y.; Ip, F.C.; Ip, N.Y.; Olean-12-eno[2,3-c] [1,2,5]oxadiazol-28-oic acid (OEOA) induces G1 cell cycle arrest and differentiation in human leukemia cell lines. PLoS One. 2013, 8, e63580.

24. Wei, J.; Liu, M.; Liu, H.; Wang, H.; Wang, F.; Zhang, Y.; Han L.; Lin, X. Oleanolic acid arrests cell cycle and induces apoptosis via ROS-mediated mitochondrial depolarization and lysosomal membrane permeabilization in human pancreatic cancer cell. .J. App.l Toxicol. 2013, 33, 756-65.

25. Wang, X.; Bai, H.; Zhang, X.; Liu, J.; Cao, P.; Liao, N.; Zhang, W.; Wang, Z.; Hai, C. Inhibitory effect of oleanolic acid on hepatocellular carcinoma via ERK-p53-mediated cell cycle arrest and mito chondrial- dependent apoptosis. Carcinogenesis. 2013, 34, 1323-1330.

26. Guo, G.; Yao, W.; Zhang, Q.; Bo, Y. Oleanolic acid suppresses migration and invasion of malignant glioma cells by inactivating MAPK/ERK signaling pathway. PLoS One. 2013, 8, e72079. 